\title{
COMPENSATION SCHEME WITH SHAPLEY VALUE FOR MULTI-COUNTRY KIDNEY EXCHANGE PROGRAMMES
}

\author{
Péter Biró, \\ Márton Gyetvai \\ Institute of Economics \\ CERS, \\ Corvinus University of Budapest \\ H-1097, Budapest, Hungary \\ E-mail: biro.peter@krtk.mta.hu, \\ gyetvai.marton@krtk.mta.hu
}

Inst. of Mathematics and Statistical Modelling

\author{
William Pettersson \\ School of Computing Science \\ University of Glasgow \\ G12-8QQ, Glasgow, United Kingdom \\ E-mail: william.pettersson@glasgow.ac.uk
}

Xenia Klimentova

INESC TEC

4200-465, Porto, Portugal

E-mail: xenia.klimentova@inesctec.pt
João Pedro Pedroso

INESC TEC,

Faculdade de Ciências

Universidade do Porto

4169-007 Porto, Portugal

E-mail: jpp@fc.up.pt

\section{KEYWORDS}

Kidney exchange programmes, integer programming, simulations, compensation scheme, Shapley value.

\begin{abstract}
Following up the proposal of (Klimentova, Viana, Pedroso and Santos 2019), we consider the usage of a compensation scheme for multi-country kidney exchange programmes to balance out the benefits of cooperation. The novelty of our study is to base the target solution on the Shapley value of the corresponding TU-game, rather than on marginal contributions. We compare the long term performances of the above two fairness concepts by conducting simulations on realistically generated kidney exchange pools.
\end{abstract}

\section{INTRODUCTION}

Currently, no cure exists for Chronic Kidney Disease (CKD). Dialysis is a common treatment for CKD, but is costly, and has poor life expectancy and quality of life for patients. Transplantation is the preferred treatment of choice, but there is a shortage of deceased donors, so the waiting lists for a deceased donor kidney are long. The other alternative for transplantation is living donation. However even if there is a willing donor for the patient, the donor and recipient may be immunologically incompatible.

A Kidney Exchange Programme (KEP) is a platform that allows incompatible recipient-donor pairs (RDPs) to exchange their incompatible donor organ for a compatible donor organ. In Europe, as surveyed in 2016 (Biró, Haase-Kromwijk, van de Klundert et al. 2019), ten countries have operated a KEP. The first was the programme of the Netherlands in 2004. In Europe, the UK programme is the largest, with an average of 135 transplants per year.
In the European KEPs matching runs are conducted at regular time intervals, typically every 3 months. Virtual crossmatch tests are used to estimate compatibility between all donors and recipients, and the results of said tests are used to determine optimal exchanges. Compatibility is represented by a directed graph, where the vertices denote the incompatible recipient-donor pairs and the directed arcs denote the potential compatible transplants between the pairs. The objective of the model is usually to maximise the number of transplants. In advanced KEPs, IP models are used to solve this problem (Abraham, Blum and Sandholm 2007).

For ethical reasons, the operations in an exchange cycle should happen simultaneously. Hence in practice logistical constraints create an upper bound on viable cycles. In Europe, the most common such bound in KEPs is 3 (e.g. the UK, Spain and Belgium), but there are examples for bound of 2 (e.g. France) and 4 (Netherlands) as well (Biró et al. 2019). Besides the number of the transplants, further considerations are given for improving the quality of the transplants or the long term success of the scheme by prioritising hard-to-match pairs, e.g. sensitive patients or patients with blood type O. A complete list of objectives in European KEPs and their possible implementations with IP techniques can be found in (Biró, van de Klundert, Manlove et al. 2020).

In some programmes, the usage of non-directed donors (NDDs) is also allowed. In most countries an NDD is an altruistic donor, who has no paired recipient, but they would offer their kidney to any recipient in the pool. Another possible NDD can be a deceased donor. In these cases, the operations can be performed consecutively. Hence these donors may initiate longer chains involving numerous pairs (Ashlagi and Roth 2014).

Larger KEP pools may result in a higher number of transplants, and merging pools can bring extra ben- 
efits due to the increased number of options. In a multi-country Kidney Exchange Programmes (mKEP) the national KEPs merge their pools. In the last few years, there several such collaborations have been established in Europe. Since 2016 the Czech Republic and Austria started a collaboration by joining the pools of two transplant centres (Böhmig, Fronek, Slavcev, Fischer, Berlakovich and Viklicky 2017). In 2018 a collaboration started between Italy, Portugal and Spain (Valentín, Garcia., Costa, Bolotinha, Guirado, Vistoli, Breda, Fiaschetti and Dominguez-Gil 2019), and Scandiatransplant has started to coordinate a joint scheme in 2019 for Sweden, Norway and Denmark (STEP Documentation 2016).

There are two types of collaboration possible for joining the pool in an mKEP. In the so-called Consecutive runs (CR) the countries first find the optimal solutions separately on their national pools; then they consider an international pool on the remaining pairs. An example for this is the Spanish-Italian-Portuguese collaboration (Valentín et al. 2019). The other possibility is the Merged pool (MP) collaboration, where the countries merge their national pools from the beginning of the optimisation process. This concept is present in the Austro-Czech and the Scandinavian programmes.

From the point of view of optimisation, an mKEP can be modelled with a similar compatibility graph, where the objective is to find the maximal number of transplants. However, in an mKEP, there are multiple countries involved, so the pairs of a cycle may belong to different countries, which may increase the logistical difficulty of the programme. Furthermore, the countries may have different constraints and objectives.

When multiple transplant centres collaborate in a regional or national KEP, it is crucial that all participants receive fair benefits. In (Ashlagi and Roth 2011), (Ashlagi and Roth 2012) the authors suggest that individual rationality (IR) of the centres should be guaranteed as a constraint in the optimisation in order to incentivise centres to reveal all of their RDPs in the programme. The constraint of iIR would guarantee that each centre gets as many transplants as they would make without the collaboration.

European mKEPs differ to US KEPs in a number of ways. In Europe the national KEPs conduct their matching runs at regular intervals, whilst the US KEPs operate on a daily basis due to the competition among the alternative programmes (Agarwal, Ashlagi, Azevedo, Featherstone and Karaduman 2018). In Europe the countries have different health care systems and various performance of their deceased programmes, so their KEPs are also different in pool sizes, their regulations and also in their distributions of RDPs, whilst in the US the pools of the transplant centres are more similar to each other. The largest national programme in the US, the National Kidney Registry, uses a scoring system to incentivise the transplant centres to fully register all of their pairs, and the registration of easyto-match pairs and altruistic donors are awarded bonus scores to ensure fairness among centres.
When the objective of the mKEP is to obtain the maximal number of transplants, the benefits from the collaboration can be unbalanced across countries. It means that some countries would proportionally increase their number of transplants less than other countries, if one considered the benefit they brought to the pool. The paper by (Klimentova et al. 2019) investigated the fairness of an mKEP by proposing the usage of a compensation scheme. The authors presented an extended MILP model and algorithm to make an mKEP fairer when running for a long period of time. The process is based on a credit system that tracks and balances out the benefits for the contributors. The authors use two fair-values for the determination of the contribution of each participant.

The Shapley value is a well-known solution concept from the field of cooperative game theory (Shapley 1953), that satisfies four important fairness axioms. An mKEP can be considered as a game with transferable utility (TU-game), where the players are the participating countries, and the value of a coalition is the number of transplants these countries can achieve together in the collaboration. Several recent articles investigated an mKEP as a cooperative game, studying the Nash-equilibria (Carvalho, Lodi, Pedroso and Viana 2017), (Carvalho and Lodi 2019) and the core (Biró, Kern, Pálvölgyi and Paulusma 2019) of the corresponding games. In this paper, we compare one of the fair-values, the Benefit value, used in (Klimentova et al. 2019) to the Shapley value. Our results show that the Shapley value provides similar effects as the Benefit value, essentially giving better outcomes for the larger countries and worse for the smaller countries, that can be seen as fairer from the point of view of their valueadding contributions. However, we found the compensation scheme with the Shapley value more stable than with the Benefit value respecting the dynamic biases from the fair values.

\section{FAIRNESS MODELS FOR MKEP}

First we describe the standard KEP model and the basic IP formulations, and then we introduce the concept of dynamic compensation schemes.

\section{Notation}

Let $D(V, A)$ denote a directed compatibility graph $D$, where $V$ corresponds to the recipient-donor pairs and $A$ corresponds to the compatible transplants. The arc $(i, j)$ represent the compatibility between the donor of pair $i$ and the recipient of pair $j$, hence the arc only exists if donor $i$ is compatible with recipient $j$. Let $\mathcal{C}$ denote the set of cycles up to length $K$. Let each cycle $c$ be a set of arcs and let $V(c)$ denote the set of vertices covered by cycle $c$.

We can include NDDs in the model as follows. For each NDD we add a vertex, which has outgoing arcs as any other vertex in the graph representing possible donations from the NDD. We also add incoming arcs to the NDD vertex from all the vertices representing RDPs. The latter dummy arcs will correspond to do- 
nations at the end of the NDD-chain that are either not performed or the donation is from the last living donor to the deceased waiting list.

In the international collaboration, let $N$ denote the set of participating countries, where the set of RDPs is partitioned according to countries as follows: $V=$ $V^{1} \cup V^{2} \cup \cdots \cup V^{|N|}$. The set of arcs $A^{n}$ denotes the arcs pointing to the pool of the country $n$, denoted by $V^{n}$. Note that $A=A^{1} \cup A^{2} \cup \cdots \cup A^{|N|}$.

\section{Model with the individual rationality constraint}

There are two types of basic IP models known in the literature for maximising the number of transplants in a KEP. One is the so-called edge-formulation, and the other one is the cycle-formulation (Abraham, Blum and Sandholm 2007). The edge-formulation considers the arcs of the graph as the variables of the model. In the cycle formulation, all of the possible cycles are enumerated, and each cycle is represented by a binary variable.

Both formulations are usable in the multi-country situation, however the cycle-formulation is usually faster to solve (Abraham, Blum and Sandholm 2007). Hence we used this formulation in our computational analysis.

As in (Klimentova et al. 2019), we also study a cycleformulation model with the IR constraint. Let $Z^{n}$ be the number of transplant that country $n$ would accomplish without any collaboration. The model $\mathcal{M}(\mathcal{C})$ considers the IR constraints in the maximisation of the number of transplants:

$$
\mathcal{M}(\mathcal{C}): \quad \max \sum_{c \in \mathcal{C}}|c| x_{c}
$$

subject to

$$
\begin{array}{cl}
\sum_{c: i \in V(c)} x_{c} \leq 1 & , \forall i \in V, \\
\sum_{c \in \mathcal{C}}|c|^{n} x_{c} \geq Z^{n} & , \forall n \in N, \\
x_{c} \in\{0,1\} \quad \forall c \in \mathcal{C} .
\end{array}
$$

Where $|c|^{n}$ denotes the number of recipients of country $n$ in cycle $c$. Constraints (IR:1) enforce each RDP to participate in at most one cycle. Constraints IR:2 is the IR constraint; they ensure that each country receives as many transplants in the mKEP as it could have achieved alone.

\section{Static fair model}

Let $\mathcal{M}^{*}$ denote the optimum of model $\mathcal{M}(\mathcal{C})$. When multiple countries start a collaboration, the model may result in an unfair solution. Some of the participants may increase the number of transplants significantly, but the increase for others may not be that large. The next model, introduced by (Klimentova et al. 2019) minimises the difference between the number of transplants and the fair-values of the countries. The fairvalue is computed with respect to the contribution of the participants. Let $\psi^{n}$ denote the fair-value of country $n$. And let $\delta^{n}$ be the absolute deviation variable, which shows the difference between the number of possible transplants and the fair-value of country $n$. Under this notation, the model is written as follows:

$$
\mathcal{F}(\mathcal{C}, \psi): \quad \min \sum_{n \in N} \delta^{n}
$$

subject to

$$
\begin{gathered}
\text { IR:1, } \text { IR:2, } \\
\sum_{c \in \mathcal{C}}|c| x_{c} \geq \mathcal{M}^{*}, \\
\delta^{n} \geq \sum_{c \in \mathcal{C}}|c|^{n} x_{c}-\psi^{n} \quad \forall n \in N, \\
\delta^{n} \geq \psi^{n}-\sum_{c \in \mathcal{C}}|c|^{n} x_{c} \quad \forall n \in N, \\
x_{c} \in\{0,1\}, \delta^{n} \geq 0 \quad \forall c \in \mathcal{C}, \forall n \in N .
\end{gathered}
$$

The objective function (1) is to minimise the absolute deviation between the real and the fair-values of each country. Constraints (3)-4 define the absolute deviations, which is $\delta^{n}=\left.\left|\psi^{n}-\sum_{c \in \mathcal{C}}\right| c\right|^{n} x_{c} \mid$. In this model, the objective is to find a solution which is as close to the fair-values as possible, without the reduction of the total number of transplants. Hence the constraint (2) guarantees that introducing fairness will not reduce the number of transplants.

\section{Dynamic fairness model}

A fair solution may not exist in a single period of an mKEP. Therefore (Klimentova et al. 2019) proposed an algorithm to guarantee the fairness of the collaboration in the long run. Let $t$ denote a period when a matching is performed in the mKEP. The programme is fair if the differences between the fair-values and the actual transplants are small in general. For the consideration of the past runs, we introduce credits for each country. When one country got less transplants in previous periods than it should, as per fair-value indications, it should get more transplant in the current period, indicated by a positive credit.

Let $\tau_{t}^{n}$ denote the target value of country $n$ in the period $t$. The target value of a country depends on the previous periods' credits $\left(c_{t}^{n}\right)$, and the fair value of the current period $\left(\psi_{t}^{n}\right)$. We call the credit as the difference between the previous periods' target value and the number of transplants obtained:

$$
c_{t}^{n}=\tau_{t-1}^{n}-s_{t-1}^{n}
$$

Where the $s_{t-1}^{n}$ denotes the number of transplants that country $n$ obtained in the previous period. Therefore the target value of the country $n$ in period $t$ can be written as:

$$
\tau_{t}^{n}=\psi_{t}^{n}+c_{t}^{n}
$$


At the beginning of an mKEP $\tau_{0}^{n}=s_{0}^{n}=0$ for all country. The algorithm differs a bit in the two types of collaborations. Let $\{\mathrm{MP}\}$ denote of the merged pools case and $\{\mathrm{CR}\}$ the case of the consecutive runs.

\section{Algorithm 1 Dynamic fairness algorithm}

Require: $\mathcal{C}$ and $\mathcal{C} \backslash$ for $n \in N$, and the credit, $c_{t}^{n}$.

1: $\quad\{\mathrm{MP} ; \mathrm{CR}\}$ Find the maximal number of transplants that the countries would conclude without the collaboration: $Z^{n}$.

2: $\{\mathrm{CR}\}$ Remove the pairs of the optimal solution of the national matching from the pools. Then create the graph for the remaining pairs.

3: $\{\mathrm{MP}\}$ Solve the $\mathcal{M}(\mathcal{C})$ for $\mathcal{M}^{*}$. In the case of $\{\mathrm{CR}\}$ it is enough to solve the model $\mathcal{M}(\mathcal{C})$ without constraint (IR:2).

4: $\{\mathrm{MP} ; \mathrm{CR}\}$ Calculate the fair-value $\psi^{n}$.

5: $\{\mathrm{MP} ; \mathrm{CR}\}$ Determine the target value: $\tau_{t}^{n}=\psi_{t}^{n}+$ $c_{t}^{n}$.

6: $\quad\{\mathrm{MP}\}$ Solve $\mathcal{F}\left(\mathcal{C}, \tau_{\sqcup}\right)$, in the $\{\mathrm{CR}\}$ case the $\mathrm{IR}: 2$ constraint is redundant.

7: $\{\mathrm{MP} ; \mathrm{CR}\}$ Save result, calculate the transplant $\left(s_{t}\right)$, determine the remaining pairs and compute the credit $c_{t}^{n}$.

\section{THE FAIR VALUES}

In the previous section, the algorithm is based on a fair allocation, with fair share $\psi^{n}$ for each country $n$. In (Klimentova et al. 2019) the authors used two different fair values, the Benefit and the Potential.

The Potential value is the number of additional transplants in an optimal mKEP that includes this country compared to the number of transplants in the mKEP if said country was excluded. The Benefit value is the result of dividing the potential value by the number of recipients within that country's individual KEP. This helps avoid situations wherein countries with larger KEPs dominate an mKEP. According to results of experiment the Benefit value provide more balanced results, so we will only consider Benefit value in this paper.

The Benefit value is similar to the Shapley value, that is well-known in the field of cooperative game theory (Shapley 1953). The Shapley value considers the payoffs of the participants by the concept of marginal contributions. The contribution of this paper is to make a comparison between the Shapley value to the Benefit value.

\section{mKEP as a game and Shapley value}

Several articles consider an mKEP as a game. The players of this game are the participating countries. The value that the players can make in any coalition $S$ is denoted by $v(S)$, and it is equal to the optimum of the model $\mathcal{M}$, when the countries of $S$ collaborate. The coalition involving all the players is called as the grand coalition. Hence it means that the value of the coalition is the maximal number of transplants with the consideration of individual rationality.

The Shapley value, introduced in (Shapley 1953), is based on the concept of the marginal contribution of the player. The improvement that the player makes in a particular ordering for the players joining the game is the marginal contribution. The Shapley value is the average of these improvements over all possible coalitions.

Let $\Theta$ denote all of the different orders in which the players can enter the game. For a $\theta \in \Theta$ order $q_{\theta}^{n}$ indicates the contribution that country $n$ adds to the coalition when she joins. Then the Shapley value can be calculated as:

$$
\psi^{n}=\frac{1}{|N| !} \sum_{\theta \in \Theta} q_{\theta}^{n}
$$

In an mKEP, $q_{\theta}^{n}$ is the increase of the transplants when the country $n$ joins to the $\theta$ ordered collaboration. In an order, $\theta$, the first country's marginal contribution is $q_{\theta}^{n_{1}}=v\left(n_{1}\right)-v(\emptyset)$. The second one's is $q_{\theta}^{n_{2}}=v\left(n_{2} \cup\right.$ $\left.n_{1}\right)-v\left(n_{1}\right)$ and the third one's is $q_{\theta}^{n_{3}}=v\left(n_{3} \cup n_{2} \cup\right.$ $\left.n_{1}\right)-v\left(n_{2} \cup n_{1}\right)$, and so on. When all of the $\theta$ orders are considered, the (7) can be simplify to the next formula:

$$
\psi^{n}=\sum_{S \subseteq(\mathcal{N} \backslash n)} \gamma_{S}[v(S \cup n)-v(S)]
$$

The $\gamma_{S}=\frac{|S| !(N-|S|-1) !}{N !}$ denotes the probability of the occurrence of the $S$ coalition. Then $(8)$ considers every possible coalition without country $n$ (even the zero-coalition, when there is no one to collaborate), and sums the effects of the inclusion of country $n$ with the weight of $\gamma_{S}$.

\section{Benefit value}

The Benefit value of the article (Klimentova et al. 2019) distributes the surplus of the coalition over the sum of the individual solutions. Hence this excess $(\sigma)$ is the value of the grand coalition minus all of the result of the separated KEPs:

$$
\sigma=v(N)-\sum_{i \in N} v(i)
$$

The ratio that the players can get from the surplus depends on their contribution to the grand coalition value. This contribution is calculated by the total improvement in the number of transplants if the country joins the others:

$$
a^{n}=v(N)-v(N \backslash n)-v(n)
$$

Therefore the Benefit payoff for the country $n$ is:

$$
\psi_{n}=v(n)+\sigma \frac{a^{n}}{\sum_{i \in N} a^{i}}
$$

In the case of $\sum_{i \in N} a^{i}=0$, the surplus is distributed equally for the players:

$$
\psi_{n}=v(n)+\frac{\sigma}{|N|} \quad \forall i
$$




\section{SIMULATION}

We used the simulator form (Santos, Tubertini, Viana and Pedroso 2017, Klimentova et al. 2019) to generate a pool of mKEP that dynamically changes over time. We investigated seven consecutive years of the mKEP, with the matching run every 90 days (4 times a year). Over the considered years the incompatible RDPs arrive, depending on the country, but stays there minimum 60 to the maximum of 2190 days, on average of 365 days uniformly. For the analysis of the data, we considered the first year as a warm-up period, to populate the pool. Hence in the statistical results, we analysed only the last six years.

We compared the results when the countries do not collaborate with the MP and CR type of collaborations. For each collaboration levels, we considered the maximisation of the number of transplants. For the latter two, we calculated the result of the fair model as well, with the Shapley and the Benefit value. We restricted the number of transplant per cycle to 3 and the number of transplants per chains initiated by NDDs to 2 .

\section{Collaboration of same sized countries.}

First, we considered a case, when three countries with similar attributes of the pools start the coalition. In this case, because the pools are identical, the contributions also should be similar. Therefore it would make the same effect for each country. Hence, in the long run, the ratio of a participant gets on average should be around $\frac{1}{3}$. We investigated this case because in this setup, with the fair models, the effect of an additional random contribution of a country can be more noticeable.

In this setup, we excluded the possibilities of NDDs. We investigated three different cases: Small, Medium and Large which refers to the sizes of the pools. In the Small case the pairs arrive on an average of 20 days, in the Medium on every ten days and for the last case, on every five days. In each case, we considered 100 randomly generated pools.

TABLE 1: Average improvement to the No-collaboration

\begin{tabular}{|l||c|c||c|c||c|c||}
\cline { 2 - 7 } \multicolumn{1}{c||}{} & \multicolumn{2}{c||}{ Small } & \multicolumn{2}{c||}{ Medium } & \multicolumn{2}{c||}{ Large } \\
\hline None & \multicolumn{2}{c||}{45.37} & \multicolumn{2}{c||}{104.76} & \multicolumn{2}{c||}{231.43} \\
\hline \hline & CR & MP & CR & MP & CR & MP \\
\hline \hline Max.Numb. & $116.66 \%$ & $124.06 \%$ & $108.40 \%$ & $114.75 \%$ & $103.37 \%$ & $108.13 \%$ \\
\hline Benefit & $116.78 \%$ & $123.38 \%$ & $108.46 \%$ & $114.57 \%$ & $103.34 \%$ & $108.20 \%$ \\
\hline Shapley & $116.85 \%$ & $123.33 \%$ & $108.47 \%$ & $114.54 \%$ & $103.37 \%$ & $108.15 \%$ \\
\hline
\end{tabular}

Table 1 presents the average results of the nocollaboration and the improvement with the different levels of collaborations and models. In every case, the collaboration resulted in an improvement in the number of transplants. Generally, the MP resulted in much higher transplants than the CR. The fair-models had a similar result in the average selected pairs, as expected.

Although the result does not change too much with the fair model, because of constraint (2), there is a tiny difference. This difference is because the alternative solution with the Benefit or Shapley value results in a different pool of remainders. As the results show, the different remaining pairs did not change the number of the selected pairs significantly. Furthermore, interestingly as the sizes of the pools increase the effect of the collaboration on the number of transplants decreases.

Comparing the bias between the target value and the concluded transplants

The result of the number of transplants was not significantly different for the Shapley and the Benefit value. To examine the differences, we investigated the bias between the target value and the concluded transplants, bias $=\tau_{t}^{n}-s_{t+1}^{n}$. The bias shows the average fairness of the model. If the average of the bias is close to zero, the participants received results that are close to their contribution. When a participant has a large bias, it means that her result from the model was far from her fair solution. When the bias is positive, the country did not get as much transplant as she has contributed since the beginning of the collaboration.

Fig. 1: biases of the models
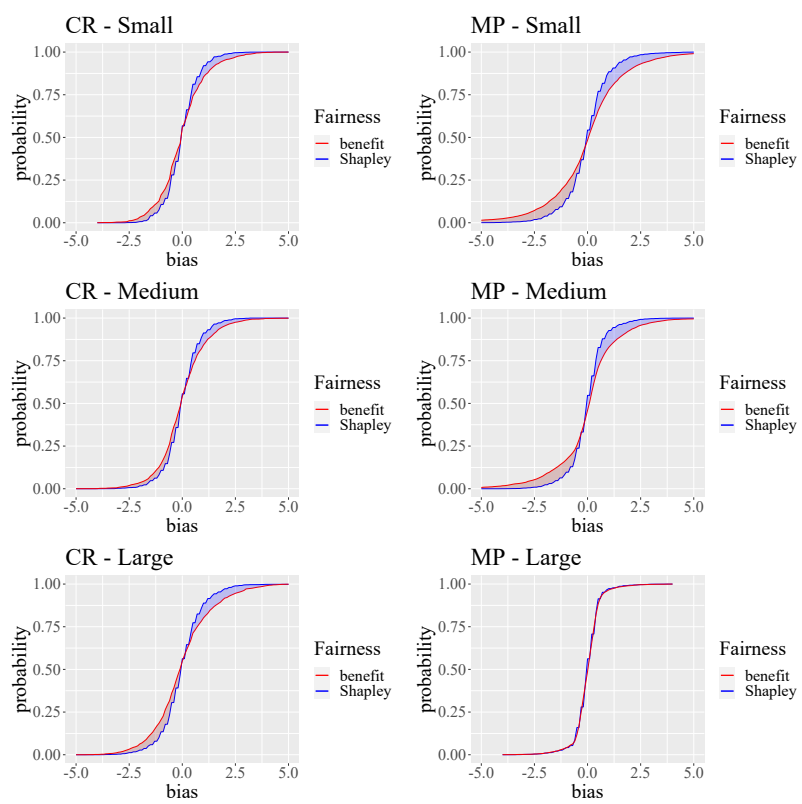

Figure 1 presents the cumulative distribution functions of the biases. The line shows the probability of the bias was less than or equal to the value of the $\mathrm{x}$ axis. The larger probabilities define the colour of the area between the two lines. In general, the Shapley value resulted in a steeper line. Therefore the biases were more concentrated near zero, than the biases of the Benefit value. The descriptive statistics of the biases can be seen in Table 2 .

In general, the bias of the model with the Shapley value resulted in a much smaller standard deviation. According to Levene's test, the difference is significant with $1 \%$ significance level for all cases, except for the Large MP case, which significantly differs only with $5 \%$ significance level. Also, the distance between the minimum and maximum were higher in almost every case with the Benefit value. Furthermore, both models resulted in a more peaked distribution, than the stan- 
TABLE 2: Descriptive statistics of the biases

\begin{tabular}{|c|c|c|c|c|c|c|}
\hline & \multicolumn{3}{|c|}{ CR } & \multicolumn{3}{|c|}{$\mathrm{MP}$} \\
\hline \multirow[t]{6}{*}{ Small } & & Benefit & Shapley & & Benefit & Shapley \\
\hline & $\min$ & -4.15 & -2.67 & $\min$ & -9.25 & -5.00 \\
\hline & $\max$ & 4.86 & 4.00 & $\max$ & 8.55 & 5.00 \\
\hline & sd & 1.11 & 0.78 & sd & 1.81 & 1.05 \\
\hline & skewness & 0.44 & 0.29 & skewness & -0.17 & 0.04 \\
\hline & kurtosis & 1.22 & 1.04 & kurtosis & 2.39 & 2.44 \\
\hline \multirow[t]{6}{*}{ Medium } & & Benefit & Shapley & & Benefit & Shapley \\
\hline & $\min$ & -5.11 & -4.00 & $\min$ & -11.79 & -4.17 \\
\hline & $\max$ & 5.69 & 3.17 & $\max$ & 11.76 & 4.67 \\
\hline & sd & 1.13 & 0.84 & sd & 1.54 & 0.84 \\
\hline & skewness & 0.27 & 0.03 & skewness & -0.56 & 0.04 \\
\hline & kurtosis & 1.61 & 1.45 & kurtosis & 5.77 & 2.94 \\
\hline \multirow[t]{6}{*}{ Large } & & Benefit & Shapley & & Benefit & Shapley \\
\hline & $\min$ & -4.53 & -3.83 & $\min$ & -4.50 & -3.83 \\
\hline & $\max$ & 5.33 & 5.67 & $\max$ & 4.07 & 4.33 \\
\hline & sd & 1.40 & 0.97 & sd & 0.61 & 0.59 \\
\hline & skewness & 0.43 & 0.31 & skewness & -0.11 & -0.09 \\
\hline & kurtosis & 0.83 & 2.19 & kurtosis & 7.48 & 6.67 \\
\hline
\end{tabular}

dard normal distribution (when kurtosis and skewness equals to 0$)$.

In summary, when the participants had similar characteristics, the results on the number of transplants were very similar for both models. However, the Shapley value resulted in a less spread result on the bias. The smaller deviation is important because it can result in a more stable mKEP.

\section{Collaboration of different sized countries.}

When the pools of the countries are not identical, their contributions to the collaboration may be quite different. In this section, we investigated the case when the size of the pools differ across countries. In this case, using a fair model is important because it would make the mKEP more beneficial to the participants with a high contribution to the shared pool.

To consider all possibilities (collaboration with larger or with smaller country), we considered an instance when three countries with different pool-sizes collaborate. We analysed a case when there is a small country with incoming pairs on 20 days average, a medium-sized country with 10 days on average and a country with a large pool where the RDPs register into the KEP on average every 5 days.

The fair models change the allocation of the results, compared to the model maximisation of the number of transplants. To investigate which participant would benefit from considering the fair values, we compared the result of the fair model to the original maximisation model without the IR constraint. For the sake of comparison of the different sized countries, we used relative changes. Therefore we calculated a relative Price of Fairness (RPoF) indicator, which shows the relative loss of the participant when the fair model is used.

Let $v$ the number of transplants that a country made by the simple maximisation model. And let the $w$ be the total number of transplants with the fair-model. Then

$$
\mathrm{RPoF}=\frac{v}{w}-1
$$

Therefore if the RPoF is positive, then the participant lost transplants when the fair model was used. Table 3 present the RPoF results of each country.

TABLE 3: RPoF of the three different sized countries.

\begin{tabular}{|c||c|c||c|c||c|c|}
\hline \multicolumn{1}{|c||}{} & \multicolumn{2}{c||}{ Small } & \multicolumn{2}{c||}{ Medium } & \multicolumn{2}{c|}{ Large } \\
\cline { 2 - 3 } & MP & CR & MP & CR & MP & CR \\
\hline \hline Benefit & $10.28 \%$ & $0.04 \%$ & $2.05 \%$ & $-0.23 \%$ & $-2.65 \%$ & $0.14 \%$ \\
\hline Shapley & $9.40 \%$ & $0.31 \%$ & $2.37 \%$ & $-0.50 \%$ & $-2.70 \%$ & $0.10 \%$ \\
\hline
\end{tabular}

When the collaboration was CR, the result of the fair-model slightly differs from the maximal number of transplants. In this case, in the first run, the set of remaining pairs the participants share, is usually much smaller than the original pool. Hence the effect of fairness is much smaller. However, in case of the collaboration is MP, then there is a much higher effect across countries. For the small-pooled country, the RPoF is higher. Therefore this country gets fewer transplants with the consideration of the Fair-value. The effect is similar but smaller for the medium-pooled country. However, the country with the largest pool got a negative RPoF. That means with the consideration of the fair-model, the large-pooled country gets more transplant than in the case of the simple maximisation of the number of transplants. In total, the results indicate that the simple maximisation of the number of transplants is not fair for the countries with larger pools. Therefore in the original model, the larger pools contribute excessively to the collaboration, but their advantage from the collaboration is smaller.

The results of the Benefit and the Shapley values slightly differ. In the case of the Shapley value, the medium-pooled country gets a somewhat fewer transplant, and the smaller one gets more.

\section{Effects of NDDs}

In the previous tests, we did not consider any NDDs. In this section, we investigate the effects when one of the participants allows NDDs in her pool. We investigated the cases where the NDDs could arrive at the pool in 180 days on average.

We again considered three country cases. Among the three countries, two have equal-sized pools, and the third one has a Large-pool. We chose this setup, to investigate the effect of the same sized pools with NDDs and the different sized pools with NDDs at the same time. With the principle of ceteris paribus, we compared three different instances: There are no NDDs; One of the small pooled countries has NDDs; the Large country has NDDs. With these instances, we would investigate the effect when a similar country, a larger one, or a smaller country, has NDDs. However, for the comparison, we calculated the results without any NDDs as well.

Table 4 presents the RPoF when no country has NDDs. The fair models in the collaboration level of CR has almost zero effect compared to the simple maximisation of the number of transplants. In the case of MP, the small countries benefited more from the 
TABLE 4: RPoF:No country has NDDs

\begin{tabular}{|c||c|c|c||c|c|c|}
\hline \multicolumn{1}{|c||}{} & \multicolumn{3}{c||}{ MP } & \multicolumn{3}{c|}{ CR } \\
\cline { 2 - 7 } & Large & Small & Small-2 & Large & Small & Small-2 \\
\hline \hline Beneit & $-2.39 \%$ & $7.94 \%$ & $5.25 \%$ & $0.32 \%$ & $0.23 \%$ & $-1.14 \%$ \\
\hline Shapley & $-2.40 \%$ & $8.39 \%$ & $5.50 \%$ & $0.31 \%$ & $0.26 \%$ & $-0.87 \%$ \\
\hline
\end{tabular}

model without fair-values. When a small pool with a larger one merges, the pairs from the smaller one has a much higher chance for transplantation. However, the pairs from the larger pool do not have that much increase. Hence the smaller countries contribution was much smaller. The large country, on the other hand, had a more significant effect on the transplants number. Therefore the country with the largest pool size would get more transplant with the fair model.

TABLE 5: RPoF: One small country has NDDs

\begin{tabular}{|c||c|c|c||c|c|c|}
\hline \multicolumn{1}{|c||}{} & \multicolumn{3}{c||}{ MP } & \multicolumn{3}{c|}{ CR } \\
\cline { 2 - 7 } & Large & Small & Small-NDD & Large & Small & Small-NDD \\
\hline \hline Beneit & $1.14 \%$ & $11.12 \%$ & $-11.69 \%$ & $0.62 \%$ & $0.63 \%$ & $-1.97 \%$ \\
\hline Shapley & $0.99 \%$ & $10.90 \%$ & $-11.05 \%$ & $0.57 \%$ & $0.43 \%$ & $-2.02 \%$ \\
\hline
\end{tabular}

In the case, when one small country can have NDDs, with the fair model, this country gets an improvement in the number of transplants. Furthermore, in this case, the large-pooled country has a slightly positive RPoF, which means it loses transplants in the fair instances. Interestingly it leads to a conclusion that even a few NDDs (registered on every half-year on average) can have more effects on the contribution to the collaboration than the size of the pool. Therefore the RPoF of the small country with NDDs became negative while the Large country's RPoF shifted positively. The other small-pooled country has even more reduction in the number of transplants compared to the original case without NDDs.

TABLE 6: RPoF:Large pooled country has NDDs

\begin{tabular}{|c||c|c|c||c|c|c|}
\hline \multicolumn{1}{|c||}{} & \multicolumn{3}{c||}{ MP } & \multicolumn{3}{c|}{ CR } \\
\cline { 2 - 7 } & Large-NDD & Small & Small-2 & Large-NDD & Small & Small-2 \\
\hline Beneit & $-4.27 \%$ & $13.44 \%$ & $8.32 \%$ & $0.24 \%$ & $1.17 \%$ & $-0.86 \%$ \\
\hline Shapley & $-4.38 \%$ & $13.11 \%$ & $8.94 \%$ & $0.13 \%$ & $0.98 \%$ & $-0.59 \%$ \\
\hline
\end{tabular}

As in Table 4, the simple maximisation model is unfair against the larger-pooled countries. Furthermore, Table 5 shows that the NDDs made more effect on the level of contribution to the collaboration than the size of the large country. Moreover, we investigated when only the largest-pooled country has NDDs. Unsurprisingly the large-pooled country with NDDs got an even more significant improvement on the number of transplants when the fair model was applied. The RPoF of this situation is shown in table 6. While in the noNDDs case, the increase of the fair model was around $2.4 \%$, with NDDs the improvement increased to around $4.3 \%$.

\section{Effects of NDDs on transplants}

Permitting NDDs in the multinational case may have a higher effect on other countries. For this, we considered every chain, initiated by a NDD and calculated the number of recipients of each country. Hence, figure 2 presents the distribution of those transplants, which concluded because of a NDD.

Fig. 2: Effects of the NDDs
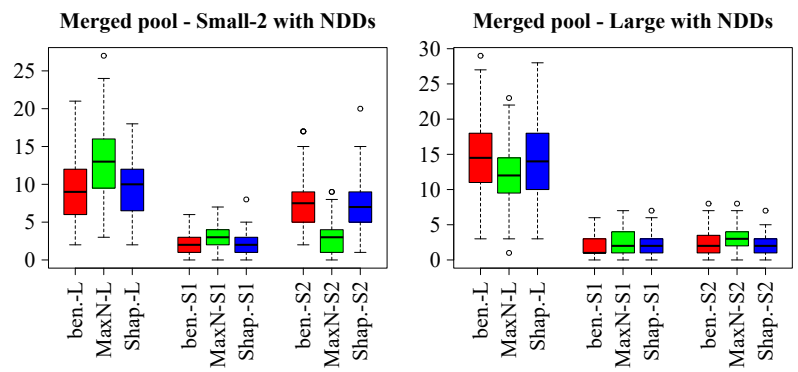

In the left side of figure 2, there is the effect of the small country's NDDs. Interestingly the NDDs resulted in more improvement for the Large country than the country of the NDDs. The fair-models somewhat reduce the difference, but still, the Large country remains the most affected. The other small country gets a bit of improvement because of the NDDs, but she also lost some transplant because of the fair values.

On the right side, there is the effect when the larger country permits NDDs. The Large country gets the most improvement in this case, similarly to the other case. Due to the provider of these donors are also the Large country, the fair values improve her results.

\section{CONCLUSION}

We concluded some tests over the Shapley value and the Benefit value. We used a multi-period simulation of an mKEP with three countries. The Shapley- and Benefit value resulted in a similar result in the number of transplants. However, the Shapley value has a slightly better result, on the bias, a.k.a the temporary unfairness of the mKEP. The bias of the Shapley value had a much smaller standard deviation in most of the cases. In general, the fair model gives an improvement of the countries with a larger pool. Hence their contribution is more relevant in the collaboration. The inclusion of NDDs mostly affected the country with larger pools. Hence when one smaller country has NDDs, they get more benefit from the fair-model.

\section{ACKNOWLEDGEMENTS}

Supported by COST Action CA15210 ENCKEP, supported by COST (European Cooperation in Science and Technology) - http://www.cost.eu/

Biró and Gyetvai were supported by the Hungarian Academy of Sciences under its Momentum Programme (LP2016-3/2018) and Cooperation of Excellences Grant (KEP-6/2018), and by the Hungarian Scientific Research Fund - OTKA (no. K129086)

\section{REFERENCES}

Abraham, D.J., A. Blum and T. Sandholm. 2007. "Clearing algorithms for Barter exchange markets: Enabling nationwide kidney exchanges." Proceedings of the 8th ACM conference on Electronic commerce pp. 295-304. 
Agarwal, Nikhil, Itai Ashlagi, Eduardo Azevedo, Clayton R Featherstone and Ömer Karaduman. 2018. Market failure in kidney exchange. Technical report National Bureau of Economic Research.

Ashlagi, I. and A.E. Roth. 2011. Individual rationality and participation in large scale, multi-hospital kidney exchange. In EC' 11 Proceedings of the 12th ACM conference on Electronic commerce. pp. 321-322.

Ashlagi, I. and A.E. Roth. 2012. "New challenges in multihospital kidney exchange." American Economic Review 102(3):354359.

Ashlagi, Itai and Alvin Roth. 2014. "Free riding and participation in large scale, multi-hospital kidney exchange." Theoretical Economics $9(3)$.

Biró, Péter, Bernadette Haase-Kromwijk, Joris van de Klundert et al. 2019. "Building kidney exchange programmes in Europe - an overview of exchange practice and activities." Transplantation 103(7):1514-1522.

Biró, Péter, Joris van de Klundert, David Manlove et al. 2020. "Modelling and optimisation in European Kidney Exchange Programmes." European Journal of Operational Research .

Biró, P., W. Kern, D. Pálvölgyi and D. Paulusma. 2019. Generalized Matching Games for International Kidney Exchange. In Proc. of the 18th International Conference on Autonomous Agents and Multiagent Systems (AAMAS 2019). pp. 413421.

Böhmig, Georg A, Jiří Fronek, Antonij Slavcev, Gottfried F Fischer, Gabriela Berlakovich and Ondrej Viklicky. 2017. "Czech-Austrian kidney paired donation: first European cross-border living donor kidney exchange." Transplant International 30(6):638-639.

Carvalho, Margarida and Andrea Lodi. 2019. "Game theoretical analysis of Kidney Exchange Programs." arXiv preprint arXiv:1911.09207.

Carvalho, Margarida, Andrea Lodi, Joao Pedro Pedroso and Ana Viana. 2017. "Nash equilibria in the two-player kidney exchange game." Mathematical Programming 161(1-2):389417.

Klimentova, X., A. Viana, J.P. Pedroso and N. Santos. 2019. "Fairness models for multi-agent kidney exchange programmes." Submitted to Omega: The International Journal of Management Science.

Santos, N., P. Tubertini, A. Viana and J.P. Pedroso. 2017. "Kidney exchange simulation and optimization." Journal of the Operational Research Society pp. 1-12.

Shapley, L. S. 1953. A Value for n-person Games. In Contributions to the Theory of Games. Annals of Mathematical Studies 28., ed. H. W. Kuhn and A. W. Tucker. Princeton, New Jersey: Princeton University Press p. 307-317.

STEP Documentation. 2016. "Scandiatransplant kidney exchange programme (STEP), Internal working document, Sahlgrenska Universitetssjukhuset." Version 1.7, January $26,2016$.

Valentín, M.O., M. Garcia., A.N. Costa, C. Bolotinha, L. Guirado, F. Vistoli, A. Breda, P. Fiaschetti and B. Dominguez-Gil. 2019. "International Cooperation for Kidney Exchange Success." Transplantation 103(6):e180-e181.

\section{AUTHOR BIOGRAPHIES}

PÉTER BIRÓ has received his $\mathrm{PhD}$ in mathematics and computer science at Budapest University of Technology in 2007 and then he was a postdoc at the Computer Science Department of Glasgow University for three years. In 2010 he joined the Institute of Economics of the Hungarian Academy of Sciences, and currently he is the head of the Momentum research group on Mechanism Design. He is also a part-time lecturer at the Department of Operations Research and Actuarial Sciences, Corvinus University of Budapest since 2013. His email address is peter.biro@krtk.mta.hu

MÁRTON GYETVAI graduated as an Actuary from the Corvinus University of Budapest in 2017 and started his $\mathrm{PhD}$ studies at the same institute in the same year, at the Doctoral School of General and Quantitative Economics. In November 2017 he joined the Mechanism Design Research Group at the Research Centre for Economic and Regional Studies. His research interest lies in the fields of Combinatorial optimization. His e-mail address is gyetvai.marton@krtk.mta.hu.

XENIA KLIMENTOVA holds a $\mathrm{PhD}$ in Operations research from Matrosov Institute for System Dynamics and Control Theory of the Siberian Branch of the RAS (2010). She is a senior researcher at the Center for Industrial Engineering and Management, INESC TEC. Her research interests lie in the field of Combinatorial Optimization. Her e-mail address is xenia.klimentova@inesctec.pt.

JOÃO PEDRO PEDROSO holds a PhD in mathematical engineering from the Université catholique de Louvain, Belgium and is presently auxiliary professor at the Faculty of Sciences, University of Porto. His research interests are on computational methods for combinatorial optimization, involving simulation and machine learning. His e-mail address is jpp@fc.up.pt.

WILLIAM PETTERSSON holds a $\mathrm{PhD}$ in Computational Graph Theory from the University of Queensland (2014). He is currently a Research Associate in the School of Computing Science at the University of Glasgow, and he studies Algorithmic Complexity, with a focus on Parameterised Complexity and Matching Problems. His email address is william.pettersson@glasgow.ac.uk.

ANA VIANA holds a PhD in Electrical and Computers Engineering from the University of Porto (2004). She is Coordinator Professor at the Polytechnic of Porto and Head of Research of the Center for Industrial Engineering and Management. Her research interests lie in the field of Combinatorial Optimization. Her e-mail address is ana.viana@inesctec.pt. 